Tropical Journal of Pharmaceutical Research January 2019; 18 (1): 101-108

ISSN: $1596-5996$ (print); 1596-9827 (electronic)

(c) Pharmacotherapy Group, Faculty of Pharmacy, University of Benin, Benin City, 300001 Nigeria.

\title{
Synergistic effects of Yiqi Huazhuo Gushen herbal formula and valsartan on metabolic syndrome complicated with microalbuminuria
}

\author{
Wang Tian-zhan ${ }^{1}$, Huo Qing-ping ${ }^{1 *}$, Wang Bing ${ }^{1}$, Wang Wen-jian ${ }^{2}$, Fu Xiao- \\ dong $^{2}$, He Yan-ming ${ }^{3}$, Wang Yu-xin ${ }^{1}$, Peng Wen-bo ${ }^{1}$, Liang Fang ${ }^{1}$, Meng Sheng- \\ $\mathrm{xi}^{1}$, Zhang Hong-yan ${ }^{1}$ \\ ${ }^{1}$ Department of Traditional Chinese Medicine, Shanghai Jiao Tong University Affiliated Sixth People's Hospital, Shanghai \\ 200233, ${ }^{2}$ Department of Integrated Traditional Chinese and Western Medicine, Huashan Hospital, Fudan University, Shanghai \\ 200040, ${ }^{3}$ Department of Endocrine, Yueyang Hospital of Integrated Traditional Chinese and Western Medicine, Shanghai \\ University of Traditional Chinese Medicine, Shanghai 200437, China
}

*For correspondence: Email: fj1169@163.com

\begin{abstract}
Purpose: To study the effects of a combination of Yiqi Huazhuo Gushen formula and valsartan on metabolic syndrome (MetS) complicated with microalbuminuria.

Methods: Patients with MetS (100), recruited from Department of the Traditional Chinese Medicine, Shanghai Jiao Tong University Affiliated Sixth People's Hospital; Department of Integrated Traditional Chinese and Western Medicine, Huashan Hospital, Fudan University; and Department of Endocrinology, Hospital of Integrated Traditional Chinese and Western Medicine in Yueyang, Shanghai University of Traditional Chinese Medicine, were randomly divided into two sets: control group $(n=50)$ given valsartan treatment, and Chinese herbal medicine (CHF) group $(n=50)$ given Yiqi Huazhuo Gushen formula in addition to valsartan. Both therapeutic regimens were given once a day for 12 weeks. The parameters measured were conversion rate of microalbuminuria (MA), ratio of urinary albumin to creatinine (UACR), 24- $h$ total volume of urinary protein (24hTP), urinary transferrin, urinary B2 microglobulin, constitutional index (CI), and waist-hip ratio (WHR). Other indices assessed were peak systolic and diastolic pressure (SBP and DBP), mean arterial blood pressure (MABP), fasting plasma glucose (FPG), postprandial 2 h blood glucose (2hPG), glycosylated hemoglobin (GH), steadystate model for insulin resistance (HOMA-IR), total cholesterol (TC), glycerin trilaurate (TG), low density lipoprotein (LDL), and high-density lipoprotein (HDL).

Results: All 100 subjects completed the clinical study. The outcome revealed that compared with 10.00 $\%$ in controls, the negative conversion of MA reached $28.00 \%$ in the CHF group $(p<0.050)$. CHF produced reductions in MA, UACR, BMI, 24hTP and urinary $\beta 2$ microglobulin $(p<0.05)$. It also led to marked increases in BMI, WHR, SBP, MAP, FPG, 2hPPG, HbA1C and HOMA-IR, and significant decreases in TG $(p<0.05)$.

Conclusion: These results suggest that CHF treatment results in alleviation of microalbuminuria and multiple cardiovascular risk factors in metabolic syndrome complicated with microalbuminuria. These effects correlate with improvements in insulin sensitivity and rectification of abnormal fat distribution.
\end{abstract}

Keywords: Metabolic syndrome, Microalbuminuria, Yiqi Huazhuo Gushen formula, Insulin resistance, Central obesity

\footnotetext{
This is an Open Access article that uses a funding model which does not charge readers or their institutions for access and distributed under the terms of the Creative Commons Attribution License (http://creativecommons.org/licenses/by/4.0) and the Budapest Open Access Initiative (http://www.budapestopenaccessinitiative.org/read), which permit unrestricted use, distribution, and reproduction in any medium, provided the original work is properly credited.

Tropical Journal of Pharmaceutical Research is indexed by Science Citation Index (SciSearch), Scopus, International Pharmaceutical Abstract, Chemical Abstracts, Embase, Index Copernicus, EBSCO, African Index Medicus, JournalSeek, Journal Citation Reports/Science Edition, Directory of Open Access Journals (DOAJ), African Journal Online, Bioline International, Open-J-Gate and Pharmacy Abstracts
} 


\section{INTRODUCTION}

Microalbuminuria (MAU) is not only an important disorder in MetS, but also a serious risk factor for angiocardiopathy [1]. Following treatment with anti-hypertensive, hypoglycemic, lipid-lowering drugs, some patients still remain MAU-positive, and the progression of renal disorder cannot be effectively controlled. This is associated with the fact that the renal damage in MetS patients is caused by a variety of risk factors which further aggravate MAU and impair kidney function [2]. Therefore, in the treatment of MetS MAU, comprehensive control and intervention for various risk factors must be strengthened so as to improve therapeutic effectiveness.

In the early stages, this research group utilized Yiqi Huaju formula to treat disorders (central obesity and non-alcoholic fatty liver) under the guidance of TCM gathering syndrome theory, with remarkable curative effects $[3,4]$. According to analogous patterns in the same disease, if MetS patients have MAU, they usually have pathological changes such as evil heat damaging kidney, internal retention of damp turbidity, and static blood-blocking collaterals. Therefore, treatment should use drugs for replenishing kidney qi and clearing collaterals based on Yiqi Huaju formula which is incorporated into Yiqi Huazhuo Gusheni formula [5].

Studies have shown that this formula can significantly improve insulin resistance in rat models of type 2 diabetes induced with highcarbohydrate, high-fat diet, and reduce urinary microalbumin [6]. In recent years, this prescription has been made into convenient granules for treating MetS-linked MAU, with improved curative effects.

\section{METHODS}

\section{Patient's profile}

Patients with MetS complicated with MAU who were recruited from Department of the Traditional Chinese Medicine, Shanghai Jiao Tong University Affiliated Sixth People's Hospital; Department of Integrated Traditional Chinese and Western Medicine, Huashan Hospital, Fudan University and Department of Endocrine; and Hospital of Integrated Traditional Chinese and Western Medicine in Yueyang, Shanghai University of Traditional Chinese Medicine, were randomly divided into two sets: control set $(\mathrm{n}=$ 50) given valsartan treatment, and Chinese herbal medicine $(\mathrm{CHF})$ group $(\mathrm{n}=50)$ given Yiqi Huazhuo Gushen formula in addition to valsartan.

\section{Diagnostic criteria}

Metabolic syndrome was diagnosed in Chinese Guidelines for Management of Dyslipidemia in Adults [7]. For positive diagnosis, the patient should have three or more of the following characteristics: (1) central obesity: waistline for men $>90 \mathrm{~cm}$, and waistline for women $>85 \mathrm{~cm}$; (2) blood TG $\geq 1.70 \mathrm{mmol} / \mathrm{L}$ (150 mg/dL); (3) blood HDL < $1.04 \mathrm{mmol} / \mathrm{L}(40 \mathrm{mg} / \mathrm{dL})$; (4) $\mathrm{BP} \geq$ $130 / 85 \mathrm{mmHg}$; (5) $\mathrm{FPG} \geq 6.1 \mathrm{mmol} / \mathrm{L}(110$ $\mathrm{mg} / \mathrm{dL}$ ) ), or $2 \mathrm{~h}$ plasma glucose after a glucose load $\geq 7.8 \mathrm{mmol} / \mathrm{L}(140 \mathrm{mg} / \mathrm{dL})$, or a history of diabetes. At the same time, the level of microalbumin/creatinine in early morning urine detected on two different days should be between 30 and $300 \mathrm{mg} / \mathrm{g}$.

\section{Ethical approval}

This study was ratified by the Ethics Committee of Traditional Chinese Medicine of the Sixth People's Hospital Affiliated to Shanghai Jiao Tong University (approval no. 20189506), and was conducted in accordance with the guidelines of Declaration of Helsinki issued in 1964 and amended in 1996 [8].

\section{Inclusion criteria}

Patients who met the above diagnostic parameters code, and who were aged from 18 to 65 years, and those who signed consent form were included.

\section{Exclusion criteria}

Patients with type 1 glycuresis or SBP greater than $180 \mathrm{mmHg}$ or DBP lower than $110 \mathrm{mmHg}$; patients with urine microalbumin/creatinine $>300$ $\mathrm{mg} / \mathrm{g}$, or 24-h urine protein $>0.5 \mathrm{~g} / 4 \mathrm{~h}$, or $\mathrm{ScR}>$ $176 \mathrm{mmol} / \mathrm{L}$; pregnant women and nursing mothers, and patients with cancer and mental illness, were excluded.

\section{Research design}

This study was stochastic, double-blinded and placebo-controlled, and was enrolled in the China Clinical Trial Registry (Registration No. ChiCTR-TRC-11001633). A total of 100 random numbers generated by Excel 2003 software were ranked in numerical order and separated equally into two groups. According to the distribution plan, random number cards were filled by a specially-assigned person and then sealed in opaque envelopes, and kept safely with blind codes. The hospital pharmacy gave drugs to patients based on the trial plan and prepared emergency letters for each participant during the 
trial. After the trial, a statistician uncovered the results blindly and counted the data. On completion of the statistics, the researchers uncovered the results again. In this trial, the participants were divided into treatment group (Yiqi Huazhuo Gushen granules plus valsartan, $80 \mathrm{mg}$ once a day) and control group (placebo plus valsartan, $80 \mathrm{mg}$ once a day), and the two groups were compared.

The sample size $(n)$ in clinical trials was calculated using Eq 1 [9].

$n=\{(\mu \alpha+\mu \beta) / \delta 2\{P 1(1-P 1)+P 2(1-P 2)\} \ldots \ldots(1)$

A $10 \%$ degree of drop-out was considered, where $\alpha=0.05, \beta=0.1$, two-sided test. The sample capacity of this research was reckoned to be at least 96 cases, while 100 cases were eventually enrolled.

\section{Treatment}

All patients were required to control their diet, do appropriate exercise, and to be consistent in taking their pre-medications such as antihypertensive, hypoglycemic, lipid-lowering therapies before enrollment.

Patients in the treatment group were given Yiqi Huazhuo Gushen granules consisting of Huangqi (Astragalus membranaceus), Huanglian (Coptis chinensis), Shengpuhuang (Pollen typhae), Zexie (the rhizome of oriental water plantain), Ludouyi (Mung bean peel), Liuyuexue (Serissa serissoides), Zhifuzi and (Radix coniti lateralis praeparata) (batch number 110502), with $23.5 \mathrm{~g}$ crude herbs per pack. The patients took the granules dissolved in warm water, twice a day, one pack a time, and the treatment course was 12 weeks. In the control group, in addition to valsartan (80 $\mathrm{mg}$ once a day), the patients were given placebo. The placebo contained the same herbs as Yiqi Huazhuo Gushen granules, but at one-twentieth of the dose in the treatment group. The herbs were made into granules with excipient and flavoring agents, and had the same color and taste as Yiqi Huazhuo Gushen granules (batch no. 110602). The method of administration and treatment course were similar to that of the treatment group.

\section{Study parameters}

\section{MAU negative conversion}

At the end of the treatment course, the criteria for evaluating MAU negative conversion were based on Chinese Expert Consensus on Screening and Intervention for Microalbuminuria in Hypertension and Diabetes Patients [10]. These were: negative conversion: urinary microalbumin/creatinine $<30$ $\mathrm{mg} / \mathrm{g}$; maintenance: $30 \mathrm{mg} / \mathrm{g} \leq$ urinary microalbumin/creatinine $\leq 300 \mathrm{mg} / \mathrm{g}$; transformation to macroalbumin: urinary microalbumin/creatinine $>300 \mathrm{mg} / \mathrm{g}$.

\section{Urine-related indicators}

Before and after treatment, early morning urine was collected from the participants so as to determine the level of microalbuminuria (MAU), urine creatinine, urinary albumin-to-creatinine ratio (UACR), urinary transferrin, urinary $\beta 2$ microglobulin, and $24 \mathrm{~h}$ total urinary protein (24hTP).

\section{Physical indictors}

Before and after treatment, the relevant physical indicators of participants were measured, including SBP, DBP, MAP, weight, height, waistline and hipline; BMI and waist-to-hip ratio (WHR) were measured.

\section{Blood glucose indicators}

The participants' BSL were taken before and after therapy. The parameters measured were FPG, 2hPPG, HbA1c), and HOMA-IR.

\section{Parameters related to blood lipids}

Blood lipid indicators were determined before and after treatment.

\section{Statistical analysis}

Data were analyzed using software SPSS16.0, and are shown as mean \pm SD. Data were analyzed by $x^{2}$ test, and grade data were tested with rank sum test. Values of $p<0.05$ were taken as indicative of statistical significance.

\section{RESULTS}

\section{General baseline patient data}

All the 100 patients with MetS-associated microalbuminuria diagnosed in out- or in-patient departments from December 2011 to December 2017 were without bias, divided into treatment and control groups, with 50 cases in each group. As shown in Table 1, there were no significant variations in gender, age, duration of disease, and baseline medications between patients in both groups $(p>0.05)$. 
Table 1: Baseline data for the patients (mean \pm SD)

\begin{tabular}{|c|c|c|}
\hline Item & $\begin{array}{l}\text { Study } \\
\text { group }\end{array}$ & $\begin{array}{l}\text { Control } \\
\text { group }\end{array}$ \\
\hline & $55.11 \pm$ & $54.97 \pm$ \\
\hline Age (years) & 8.96 & 8.39 \\
\hline Sex (male/female) & $27 / 23$ & $28 / 22$ \\
\hline Smoking history (n) & 25 & 27 \\
\hline Drinking history $(\mathrm{n})$ & 13 & 13 \\
\hline $\begin{array}{l}\text { Family history of diabetes } \\
\text { (n) }\end{array}$ & 41 & 36 \\
\hline Diabetes (n) & 47 & 49 \\
\hline $\begin{array}{l}\text { Duration of diabetes } \\
\text { (years, } \bar{x} \pm \mathrm{sd} \text { ) }\end{array}$ & $\begin{array}{c}11.24 \pm \\
5.91\end{array}$ & $\begin{array}{l}11.16 \pm \\
6.06\end{array}$ \\
\hline \multicolumn{3}{|l|}{ Hypoglycemic drugs (n) } \\
\hline Biguanides & 40 & 38 \\
\hline Sulfonylureas & 23 & 21 \\
\hline $\begin{array}{l}\text { Alpha-glucosidase } \\
\text { Inhibitors }\end{array}$ & 39 & 38 \\
\hline Hypertension (n) & 48 & 47 \\
\hline \multicolumn{3}{|l|}{ Antihypertensive drugs ( $n$ ) } \\
\hline $\begin{array}{l}\text { Angiotensin II receptor } \\
\text { antagonists }\end{array}$ & 50 & 50 \\
\hline Calcium antagonists & 40 & 39 \\
\hline Diuretics & 8 & 7 \\
\hline Beta-blockers & 2 & 3 \\
\hline \multicolumn{3}{|l|}{ Dyslipidemic agents ( $\mathrm{n}$ ) } \\
\hline Statins & 33 & 28 \\
\hline Fibrates & 10 & 9 \\
\hline
\end{tabular}

MAU negative conversion

MAU negative conversion in treatment group was significantly higher than that in the control group $\left(28.00 \%\right.$ vs $\left.10.00 \% ; x^{2}=10.048, p<0.01\right)$ (Table 2).

\section{Urine-related indicators}

Table 3 shows that there were obvious disparities in the levels of MA, UACR, 24hTP and urinary $\beta 2$ microglobulin before and after treatment in the treatment group $(p<0.05)$. In the control group, MA, UACR and urinary $\beta 2$ microglobulin before treatment differed significantly from the corresponding values posttreatment $(p<0.05)$. Moreover, after treatment, there were significant differences in the levels of MA, UACR, 24hTP and urinary $\beta 2$ microglobulin levels both groups $(p<0.05)$. These results are depicted in Table 3.

\section{Changes in physical indicators}

Marked differences between the pre- and posttreatment values of BMI, WHR, SBP and MAP were seen in the treatment group $(p<0.05)$. In addition, there were marked differences in between the pre- and post-treatment levels of SBP and MAP in the control group $(p<0.05)$. After treatment, the two groups differed significantly as regards BMI, WHR, SBP and MAP levels $(p<0.05)$. These results are displayed in Table 4.

Table 2: Degree of MAU negative conversion $(N=50)$

\begin{tabular}{lcccc}
\hline Group & $\begin{array}{c}\text { Negative } \\
\text { conversion }\end{array}$ & Maintenance & Transformation (\%) & $\begin{array}{c}\text { Negative } \\
\text { conversion (\%) }\end{array}$ \\
\hline Treatment & 14 & 33 & 3 & 28.00 \\
Control & 5 & 40 & 5 & 10.00 \\
\hline
\end{tabular}

Table 3: Changes in urine related indicators (mean \pm SD)

\begin{tabular}{lcccccc}
\hline Group & Time & MA (mg/L) & UACR $(\mathbf{m g} / \mathbf{g})$ & $\begin{array}{c}\mathbf{2 4 h T P} \\
\mathbf{( g / 2 4 h})\end{array}$ & $\begin{array}{c}\text { Urinary } \\
\text { transferrin } \\
(\mathbf{m g} / \mathbf{L})\end{array}$ & $\begin{array}{c}\text { Urinary } \boldsymbol{\beta 2} \\
\text { microglobulin } \\
\text { (mg/L) }\end{array}$ \\
\hline \multirow{2}{*}{ Study } & Before & $99.30 \pm 76.14$ & $114.89 \pm 73.05$ & $0.14 \pm 0.06$ & $6.05 \pm 6.57$ & $0.74 \pm 0.72$ \\
& After & $46.82 \pm 36.79^{* \#}$ & $62.78 \pm 48.03^{* \#}$ & $0.07 \pm 0.05^{* \#}$ & $3.19 \pm 3.24$ & $0.40 \pm 0.42^{* \#}$ \\
\multirow{2}{*}{ Control } & Before & $99.06 \pm 71.01$ & $108.60 \pm 80.87$ & $0.13 \pm 0.07$ & $5.91 \pm 7.15$ & $0.77 \pm 0.73$ \\
& After & $83.36 \pm 79.88^{*}$ & $91.02 \pm 73.57^{*}$ & $0.11 \pm 0.06$ & $4.48 \pm 7.06$ & $0.61 \pm 0.54^{*}$ \\
\hline
\end{tabular}

${ }^{*} P<0.05$, relative to same group prior to the treatment; $\# p<0.05$, relative to control group post-treatment

Table 4: Changes in physical indicators (mean \pm SD)

\begin{tabular}{lllllll}
\hline Group & Time & BMI $\left(\mathbf{k g} / \mathbf{m}^{2}\right)$ & WHR & SBP $(\mathbf{m m H g})$ & DBP $(\mathbf{m m H g})$ & MAP $(\mathbf{m m H g})$ \\
\hline \multirow{2}{*}{ Study } & Before & $27.81 \pm 2.11$ & $0.95 \pm 0.060$ & $138.44 \pm 9.29$ & $82.24 \pm 8.19$ & $101.10 \pm 7.10$ \\
& After & $24.34 \pm 0.43^{* \#}$ & $0.87 \pm 0.05^{* \#}$ & $125.91 \pm 7.12^{* \#}$ & $78.47 \pm 5.35$ & $94.28 \pm 5.28^{* \#}$ \\
\cline { 2 - 6 } Control & Before & $27.73 \pm 2.57$ & $0.95 \pm 0.059$ & $140.32 \pm 10.24$ & $82.46 \pm 7.00$ & $101.80 \pm 6.36$ \\
& After & $27.61 \pm 2.75$ & $0.94 \pm 0.048$ & $130.56 \pm 7.99^{*}$ & $79.88 \pm 5.43$ & $96.84 \pm 4.65^{*}$
\end{tabular}

${ }^{\star} P<0.05$, compared to the same group prior to treatment; $\# p<0.05$, compared with the control group after treatment 
Table 5: Changes in blood glucose indicators (mean \pm SD)

\begin{tabular}{llcccc}
\hline Group & & FPG (mmol/L) & $\begin{array}{c}\text { 2hPPG } \\
\text { (mmol/L) }\end{array}$ & HbA1c (\%) & HOMA-IR \\
\hline Study group & Before treatment & $8.20 \pm 2.42$ & $12.43 \pm 3.79$ & $8.10 \pm 1.49$ & $4.41 \pm 1.24$ \\
$(n=50)$ & After treatment & $6.23 \pm 1.31^{*} \#$ & $8.68 \pm 2.56^{* \#}$ & $7.04 \pm 1.26^{* \#}$ & $2.38 \pm 1.92^{* \#}$ \\
Control group & Before treatment & $8.18 \pm 2.46$ & $12.14 \pm 3.74$ & $8.06 \pm 1.38$ & $4.37 \pm 1.83$ \\
$(n=50)$ & After treatment & $7.06 \pm 1.41^{*}$ & $10.62 \pm 3.24^{*}$ & $7.52 \pm 1.36^{*}$ & $3.54 \pm 1.88^{*}$ \\
\hline
\end{tabular}

${ }^{\star} P<0.05$, compared to the same group prior to treatment; $\# p<0.05$, compared with the control group after treatment

Table 6: Blood lipid indicators

\begin{tabular}{llcccc}
\hline Group & Time & TC & TG & HDL-C & LDL-C \\
\hline \multirow{2}{*}{ Study } & Before treatment & $4.85 \pm 0.76$ & $2.26 \pm 0.51$ & $1.06 \pm 0.28$ & $3.11 \pm 0.85$ \\
& After treatment & $4.24 \pm 0.78$ & $1.62 \pm 0.58^{*}$ & $1.19 \pm 0.27$ & $2.54 \pm 0.71$ \\
\cline { 2 - 5 } Control & Before treatment & $4.79 \pm 0.56$ & $2.20 \pm 0.51$ & $1.07 \pm 0.32$ & $3.08 \pm 0.76$ \\
& After treatment & $4.25 \pm 0.68$ & $1.88 \pm 0.44$ & $1.12 \pm 0.21$ & $2.55 \pm 0.52$ \\
\hline${ }^{*} P<0.05$ & compared to the & &
\end{tabular}

${ }^{*} P<0.05$, compared to the same group before treatment; $\# p<0.05$, compared with the control group after treatment

\section{Blood glucose indicators}

The results in Table 5 show that FPG, 2hPPG, $\mathrm{HbA} 1 \mathrm{c}$ and HOMA-IR in differed significantly between patients in both groups, before and after treatment $(p<0.05)$.

\section{Blood lipid indicators}

As shown in Table 6, TC, TG, LDL-c and HDL-C levels in the treatment group differed significantly from those in the control group $(p<0.05)$. Moreover, TG level differed significantly between the control group and treatment groups after treatment $(p<0.05)$. Blood lipid indicators (mean $\pm \mathrm{SD}, \mathrm{mmol} / \mathrm{L})$.

\section{DISCUSSION}

Increases in the prevalence of MetS (characterized by central obesity, hyperglycemia, hyperlipidemia, and hypertension) are associated with enhanced risks of diabetes and cardiovascular disease, and rapid increases in incidence of renal disease [11]. Microalbuminuria (MAU) is an important symptom of MetS. It is not only a sensitive indicator for diagnosing early diabetic nephropathy and other causes of kidney damage, but also a sign of extensive vascular dysfunction [12]. Due to the multiplicity of factors that impair kidney function, MetS patients have higher incidence of MAU than patients with simple diabetes or hypertension, and their kidneys are subjected to more threats.

Patients with type 2 diabetes and MetS have incidence of MAU-positive almost $14 \%$ higher than type 2 diabetic patients without MetS, and the prevalence of MAU rises with augmentation of MetS components [10]. Moreover, MAU is a strong factor for predicting cardiovascular ailments. Clinical studies have revealed that the risks of carotid intima thickening, left ventricular hypertrophy, ischemic cardiac events, and peripheral vascular disease are much higher in hypertension and/or diabetics with MAU than in people without MAU [13]. Thus, early prevention and treatment of MAU is of great significance in preventing and delaying the development of MetS-associated kidney damage, thereby ultimately preventing cardiovascular disease.

In this clinical trial, the participants were routinely treated with Western medicines including hypoglycemic, hypotensive, and lipid-lowering drugs before enrollment, but even if their blood glucose, blood pressure and blood lipids were partially regulated, they had MAU as before. This suggests that apart from the common controllable aspects including blood pressure, blood glucose, and blood lipids, the renal function of MetS people with MAU may be impaired by factors that are not fully recognized or effectively controlled, the so-called "residual risks". Therefore, strengthening the management of "residual risks" can further improve the effectiveness of treatment.

The basic pathological link to MetS is insulin resistance [14]. Insulin resistance and its accompanying hyperinsulinemia can result in high filtration, high perfusion, high pressure and high aggregation of glomeruli [15]. Hyperinsulinemia dilates afferent arteries, elevates glomerular filtration rate, enhances renal plasma flow and changes renal hemodynamics, leading to glomerular hyperfiltration, high perfusion, and high pressure. In addition, it can directly affect the kidneys, promote sodium retention, elevate systemic blood pressure, and further augment glomerular internal pressure, thereby aggravating kidney 
damage. It can also damage vascular endothelium, stimulate the generation of endothelial cell plasminogen activator inhibitor (PAI-1), and cause hypercoagulable state, thus aggravating vascular lesions, and impairing the renal function by releasing inflammatory factors and inhibiting the renin-angiotensin system [16].

Individuals with central obesity are at high risk of developing MAU, since the former is linked to resistance to insulin [17]. Therefore, enhancing the sensitivity of insulin and improving central obesity are important ways to reduce MetSassociated MAU, for which treatment there are no ideal drugs in Western medicine. Rosiglitazone, a representative of insulin sensitizers, may increase the danger of angiocardiopathy (including myocardial infarction, stroke, and heart failure), which strictly restricts its use [18]. Traditional Chinese medicine (TCM) has a good prospect in this respect.

In this clinical trial, TCM theory and the modern medical knowledge of MetS were combined to propose the innovative idea of gathering syndrome and analogous patterns in the same disease. Insulin resistance in MetS is seen in TCM as non-transformation due to spleen deficiency. Congenital deficiency or large food intake and lack of exercise damage organ $q i$ transformation, especially spleen qi transformation, so that spleen qi fails to distribute essence, and essential qi is unable to flow, and food cannot be transformed, resulting in glucose or lipid metabolism disorder. Thus, lipids accumulate in the abdomen leading to the development of central obesity. Furthermore, blood lipids, blood glucose, and blood pressure are elevated, and visceral fat infiltration become transformed into pathological factors such as evil heat, dampness and turbidity, and blood stasis which further damage target organs [19].

With respect to MetS patients with MAU, according to the idea of "same disease with similar syndrome", the patients have "spleen deficiency" and the common pathogenesis of "gathering syndrome", and also have similar syndromes such as kidney-damaging evil heat, internal retention of turbidity, and blood stasis blockage of collaterals. Thus, Ludouyi and Liuyuexue were added to clear heat and drain dampness, while Fupian was used to warm yang and supplement the kidney based on Yiqi Huaju formula. With cold and warm drugs, this formula is known as Yiqi Huazhuo Gushen formula [20]. The granules used in this study were made from this formula.
Cytological experiments were conducted on some ingredients in Yiqi Huazhuo Gushen formula, and it was found that astragalan, berberine and total flavonoids of Pollen typhaecan acted on some signal transduction pathways such as phosphatidylinositol 3-kinase or $\beta$-arrestin-2, enhanced the sensitivity of insulin, and increased the utilization of glucose by cells [21 - 25]. In previous animal experiments, it was observed that Yiqi Huazhuo Gushen formula also has insulin-sensitization effect in diabetic rat models, which conforms to the findings in this study [26]. Combined with results of previous studies, it can be seen that Yiqi huazhuo Gushen formula significantly improves abnormal distribution of lipids and increases insulin sensitization.

The BP of individuals with MetS should be regulated to $130 / 80 \mathrm{mmHg}$ or even lower. In this study, the patients in both groups took antihypertensive drugs before enrollment, but most of them had abnormal blood pressure (higher than $140 / 90 \mathrm{mmHg}$ ). After treatment, the SBP and mean arterial blood pressure of both groups were markedly reduced, with the treatment group having a greater reduction in blood pressure. In another study, a positive correlation between multiple blood pressure parameters in patients with metabolic syndrome and insulin resistance was confirmed [27]. Therefore, it may be inferred that Yiqi Huaju granule is effective in controlling blood pressure in MetS patients with MAU, due most probably to insulin sensitization.

The findings of this study reveal that the MAU negative conversions were 28.00 and $10.00 \%$ in the treatment and control patients, respectively. The treatment group had better MAU negative conversion than the control group. In terms of urine-related indicators (MA, UACR, 24hTP, and urinary $\beta 2$ microglobulin); physical signs (BMI, WHR, SBP, and MAP); blood glucose indicators (FPG, 2hPPG, HbA1c, and HOMA-IR), and blood lipids (TG), improvements in treatment patients were superior to those in control patients.

\section{Limitations of the study}

A small number of samples was used in this study. It is necessary to enlarge the sample size in future investigations, and study the link amongst Yiqi Huazhuo Gushen formula, metabolic syndrome and microalbuminuria.

\section{CONCLUSION}

The use of combination of Yiqi Huazhuo Gushen formula and valsartan for treating metabolic 
syndrome-linked MAU effectively controls microalbuminuria progression, improves insulin sensitivity, reduces the degree of central obesity, and comprehensively reverses several cardiovascular risks of MetS. Thus, this combination treatment merits clinical application.

\section{DECLARATIONS}

\section{Acknowledgement}

This research was supported by Shanghai Health and Family Planning Commission Chinese Medicine Research Fund (no. 2018LQ011); Shanghai Sixth People's Hospital Pre-Research Fund (no. LYNYLC-0091); Project of National Cerebrated Traditional Chinese Medicine Expert Wang Wen-Jian Inheritance Studio (no. MLZJGZS-2017002); Shanghai Science and Technology Committee Medical Guidance Program (no. 12401905100); Shanghai Science And Technology Committee Medical Guidance Program (no. 16401934200); Shanghai Integrated Chinese and Western Medicine Clinical Cultivation Project (no. ZY3-LCPT-22007); Association of Integrative Chinese and Western Medicine, Shanghai (no. zxyQ-1245); and Shanghai TCM Clinical Advantage Specialty Construction Project (no. ZYSNKD-YLYSZK013).

\section{Conflict of interest}

There is no conflict of interest in this subject.

\section{Author contributions}

We state that this subject was done by the author(s) named in this article and all claims and debts relevant to the content of this article will be borne by the authors. The manuscript was read and approved for publication by all the authors. Wang Wenjian, Wang Tianzhan and Huoqingping conceived and designed the study. Wang Tian-zhan, Huo Qing-ping, Wang Bing, Wang Wen-jian, Fu Xiao-dong, He Yan-ming, Wang Yu-xin, Peng Wen-bo, Liang Fang, Meng Sheng-xi, Zhang Hong-yan collected and analyzed the data, while Wang Tian-zhan wrote the manuscript.

\section{REFERENCES}

1. Ozyol A, Yucel O, Ege MR, Zorlu A, Yilmaz MB. Microalbuminuria is associated with the severity of coronary artery disease independently of other cardiovascular risk factors. Angiol 2012; 63(6): 457-460.
2. Ninomiya T, Kiyohara Y. Albuminuria and chronic kidney disease in association with the metabolic syndrome. J Cardiometab Syndr 2007; 2(2): 104-107.

3. He CY, Wang WJ, Li B, Xu DS, Chen WH, Ying J, He YM. Clinical research of Yiqi Sanju Formula in treating central obese men at high risk of metabolic syndrome. $J$ Chin Integr Med 2007; 5(3): 263-267.

4. Lou SY, Liu Y, Ma YY, Chen HY, Chen WH, Ying J, He YM, Wang WJ. Effects of Yiqi Sanju Formula on nonalcoholic fatty liver disease: a randomized controlled trial. J Chin Integr Med 2008; 6(8): 793-798.

5. Wang WJ. Analogous syndrome in the same disease and treatment based on differentiation of disease of TCM thinking on TCM development by learning scientific outlook on development. World Sci Technol Mod Tradit Chin Med 2011; 13(2): 226-231.

6. Xue HL, Zhang Z, Wang WJ, Chen WH, Ying J. Effects of Yiqi Zengming formula on microalbuminuria and insulin resistance of type 2 diabetes rats. Chin J Gerontol 2011; 31(21): 4213-4215.

7. Joint Committee issued Chinese guidelines for the management of dyslipidemia in adults. Chinese Guidelines for Management of Dyslipidemia in Adults.Chin J Cardiol 2016; 44(10): 833-853.

8. World Health Organization. Declaration of Helsinki. $\mathrm{Br}$ Med J 1996; 313(7070): 1448-1449.

9. Jin BH, Deng W. Clinical trials. Shanghai: Fudan Univ Press2004; 33(2): 83-84.

10. Sun NL. Interpretations on Chinese Expert Consensus on Screening and Intervention for Microalbuminuria in Hypertension and Diabetes Patients. Chin $J$ Hypertension 2012; 20(6): 515-517.

11. Sowers JR. Metabolic risk factors and renal disease. Kidney Int 2007; 71(8): 719-720.

12. Khosla N, Sarafidis PA, Bakris GL. Microalbuminuria. Clin Lab Med 2006; 26(3): 635-653.

13. Esteghamati A, Rashidi $A$, Khalilzadeh $O$, Ashraf $H$, Abbasi M. Metabolic syndrome is independently associated with microalbuminuria in type 2 diabetes. Acta Diabetol 2010; 47(2): 125-130.

14. Vaur L, Gueret $P$, Lievre $M$, Chabaud S, Passa $P$. Development of congestive heart failure in type 2 diabetic patients with microalbuminuria or proteinuria: observations from the DIABHYCAR (type 2 diabetes, Hypertension, Cardiovascular Events and Ramipril) study. Diabetes Care 2003; 26(3):855-860.

15. Parvanova Al, Trevisan $R$, Iliev IP, Dimitrov $B D$, Vedovato $M$, Tiengo $A$, Remuzzi $G$, Ruggenenti $P$. Insulin resistance and microalbuminuria: a crosssectional, case-control study of 158 patients with type 2 diabetes and different degrees of urinary albumin excretion. Diabetes 2006; 55(5): 1456-1462.

16. Henegar JR, Bigler SA, Henegar LK, Tyagi SC, Hall JE. Functional and structural changes in the kidney in the early stages of obesity. J Am Soc Nephrol 2001; 12(6): 1211-1217. 
17. Locatelli F, Pozzoni P, Del VL. Renal manifestations in the metabolic syndrome. J Am Soc Nephrol 2006; 17(2): 81-85.

18. Hernandez AV, Usmani A, Rajamanickam A, Moheet A. Thiazolidinediones and risk of heart failure in patients with or at high risk of type 2 diabetes mellitus: a metaanalysis and meta-regression analysis of placebocontrolled randomized clinical trials. Am J Cardiovasc Drugs 2011; 11(2): 115-128.

19. Wang WJ, Liu Y. Analysis on "non-transportation due to spleen deficiency" and "non-transformation due to spleen deficiency". J Shanghai Univ Tradit Chin Med 2013; 27(1): 8-10.

20. Wang WJ, Shen ZY. From "Different Patterns in the Same Disease" to "Analogous Patterns in the Same Disease". Chin J Integr Trad West Med 2014; 34(1): 912.

21. Wang SH, Wang WJ, Wang XF, Chen WH. Effects of astragalan and berberine on glucose metabolism and cell differentiation of 3T3-L1 adipocytes. Chin $J$ Integr Trad West Med 2004; 24(10): 926-928.

22. Liu Y, Lou SY, He YM, Chen WH, Ying J, Wang WJ, et al. Effects of berberine on protein expression and gene PPARY and C/EBPa mRNA related to the proliferation and differentiation of 3T3-L1 pre-adipocytes proliferation and differentiation. Chin J Integr Trad West Med 2008; 28(11): 1005-1009.

23. He YM, Wang WJ, Chen WH, Ying J. Effects of total flavonoids of Pollen typhae on mRNA gene expression of peroxisome proliferator-activated receptor family in 3T3-L1 adipocytes. J Chin Integr Med 2008; 6(9): 939941.

24. Feng $X T$, Wang $T Z$, Chen $Y$, Chen WH, Ying J, Wang WJ. Pollen Typhae total flavone improves insulininduced glucose uptake through the beta-arrestin-2mediated signaling in $\mathrm{C} 2 \mathrm{C} 12$ myotubes. Int $\mathrm{J} \mathrm{Mol} \mathrm{Med}$ 2012; 30(4): 914-922.

25. Feng $X T$, Wang $T Z$, Leng J, Chen $Y$, Liu JB, Liu Y, Wang WJ. Palmitate contributes to insulin resistance through downregulation of the Src-mediated phosphorylation of Akt in C2C12 myotubes. Biosci Biotechnol Biochem 2012; 76(7): 1356-1361.

26. Zhang Z, Xue HL, Liu Y, Wang WJ. Yi-Qi-Zeng-MinTang, a Chinese medicine, ameliorates insulin resistance in type 2 diabetic rats. World $\mathrm{J}$ Gastroenterol 2011; 17(8): 987-995.

27. Chen Y, Fu DY, Fu XD, He YM, Wang WJ. Clinical study on Yiqi Huaju formula combined with routine therapy for hypertension of metabolic syndrome. Chin J Integr Trad West Med 2014; 34(6): 680-687. 